Gynäk. Rdsch. 1970;9:I-VIII

\title{
Contents, Vol. 9, 1970
}

\section{Übersichtsaufsätze}

Audet-Lapointe, P. und Vauclair, R. (Montreal): Die Granulosatumoren. Literaturübersicht und Beschreibung von 7 Fallen 249

Janovski, N. A. (Würzburg): Dysplastische und prämaligne Veränderungen der Vulva. Histologische und histochemische Betrachtung 161

KURZFASSUNGEN VON ZEITSCHRIFTENARTIKELN

Adams, Ruth Ann : vide Keeve, J. Ph.

Alan, B.; Ng, A. und Reagan, J. W. (Cleveland, Ohio): Über das mikroinvasive

Karzinom der Zervix 177

Anderson, G. V.: vide Pearson, H. E.

Antolak, S. J., Jr.; Smith, J. P. und Doolittle, K. H. (Columbus, Ohio): Hypo-

spadiebildung bei der Frau 59

Arthes, F. G.: vide Sartwell, P. E.

Asire, A. J.: vide Cutler, S. J.

Asscher, A. W.: vide Sussmann, M.

Atchley, C. (Oxford, Ohio): Auskunftsverweigerung und Mitarbeit bei einer

Reihenuntersuchung pensionierter Frauen: Eine Analyse ausgewählter Charakteristika 230

Austin, J. H.: vide Macmahon, B.

A very, G. B.; Kraybill, E. N. und Mullick, U. C. (Washington, DC): Unter-

suchungen des Neugeborenen 216

Baden, M.: vide Mamunes, P.

Badib, A. O.; Kurohara, S. S. und Webster, J. H. (Buffalo, N.Y.): Strahlen-

therapie beim Zervixkarzinom der alten Frau186

Bass, J. W.: vide Mamunes, P. Bech, I.: vide Fischerman, K. Bernstine, L. R. (Bethesda, Md.):

Studien über die Sicherheit der Ultraschall-

Untersuchung (Doppler-Prinzip) bei klinischer Anwendung und in der Gewebs-

kultur 243

Bertrand, H.: vide Raby, C. Bhushan, Vidya: vide Prasad, B. G.

Blacker, C. P. und Peel, J. H. (London): Sterilisation von Frauen 7

Bonnar, J.; McNicol, G. P. und Douglas, A. S. (Glasgow): Blutgerinnung und

Fibrinolyse intra et post partum 280

Bourgeois, C. H.: vide Gall, S. A.

Brish, M.: vide Hack, M.

Britt, R. P.: vide Voigt, J. C.

Brown, D. W.: vide Niehoff, R. D.

Buchsbaum, H. und Caruso, Ph. A. (Brooklyn, N.Y.): Schusswunden des schwangeren Uterus 291

Burt, D.: vide Emery, A. E. H. Cade, J. F.: vide Hirsh, J. 
Inhaltsverzeichnis

III

Caldeyro-Barcia, R. (Uruguay): Symposium über uterushemmende Pharmaka

und deren Wirkung auf die Mutter, den Fetus und das Neugeborene. Punta del

Este, Uruguay, 22.-25. März 1970

19

Campbell, H.: vide Sussmann, M.

Caruso, Ph. A.: vide Buchsbaum, $\mathrm{H}$.

Chenudet, P.: vide Mahon, R.

Chernick, V.: vide Pusey, V. A.

Cohen, M. B.: vide Wenner, Naomi K.

Cohen, B. H. und Sayre, J. E. (Baltimore, Md.): Weitere Beobachtungen über die Beziehungen

zwischen mütterlichen ABO- und Rh-Blutgruppen und fetalem Tod 125

Conolly, D. P.: vide Fracchia, A. A.

Connell, E.: vide Reyniak, J. V.

Crapanzano, T. J.: vide McCann, St. W.

Curran, W. J. (Boston, Mass.): Illegale therapeutische Aborte: das Dilemma unse-

rer Zeit212

Cutler, S. J.; Zippin, C. und Asire, A. J. (San Francisco, Cal.): Die prognostische

Bedeutung palpabler Lymphknoten beim Brustkrebs101

Davay, D. A.: vide Macintosh, I. J. C.

Del Gaizo, A.: vide Kamat, M. H.

Diers, C. J.: vide Amundsen, D. W.

Donald, J.: vide Hellman, L. M.

Doolittle, K. H.: vide Antolak, S. J., jr.

Dubeco, J. P.: vide Mahon, R.

Dufuss, G. M.: vide Hellman, L. M.

Douglas, A. S.: vide Bonnar, J.

El-Mahallawi, M. N.: vide Fadel, H. E.

Emery, A. E. H.; Nelson, M. M.; Gordon, G., and Burt, D. (Edinburgh): Prä-

natale Diagnose 15

Evans, J.: vide Kneale, B.

Evans, J. A. S.: vide Sussmann, M.

Evans, K. T.: vide Sussmann, M.

Fadel, H. E.; Sabour, M. S.; Mahran, M.; Seif El-Din, D. und El-Mahallawi, M. N. (Cairo):

Nachweis der Rückbildung der Nierenläsion und der Nieren-funktionsstörung bei

Schwangerschaftstoxikosen anhand der Nierenbiopsie . . 208

Farrow, J. H.: vide Fracchia, A. A.

Fearing, J. M.: vide Wenner, Naomi K.

Fischerman, K.; Bech, I.; Foged, P.; Hostrup-Pedersen, J. und Lauritzen, J. B.

(Kopenhagen): Die Bedeutung der aktiven chirurgischen Behandlung von

Mammatumoren bei der Frühdiagnose von Brustkrebs $\quad 110$

Fischerman, K.; Bech, I.; Foged, P.; Hostrup-Pedersen, J. und Lauritzen, J. P.

(Kopenhagen): Beziehung zwischen zystischer Fibroadenomatose und Brustkrebs 112

Fletcher, G. H.; Montague, E. D. und White, E. C. (Huston, Tex.): Die Be

deutung der Bestrahlung der peripheren Lymphknoten in Verbindung mit der

radikalen Mastektomie vvegen Brustkrebses 103 
Florman, A. L. und Teubner, D. (Manhasset, NY): Anregung von Bakterienwachstum durch Mekonium im Fruchtwasser 28

IV

Inhaltsverzeichnis

Foged, P.: vide Fischerman, K.

Forrest, A. P. M.; Henk, E. N. und Gravelle, I. H. (Cardiff): Die Behandlung

des Brustkrebses im Frühstadium 95

Fowler, W. C: vide Shingleton, H. M.

Fracchia, A. A.; Farrow, J. H.; Palo, A. J. De; Conolly, D. P. und Huvos, A.G. (New York, N.Y.): Die Kastration beim primär inoperablen oder rezidivierenden Mammakarzinom 114

Friedman, St. und Goldfien, A. (San Francisco, Calif.): Amenorrhoe und Galaktorrhoe nach oralen Kontrazeptiva 310

Frisancho, A. R.; Garn, St. M. und Rohmann, Ch. G. (Yellow Springs, Ohio):

Die neue Methode zur Bestimmung des Menarche-Alters 67

Früherkennung des Brustkrebses, Die: Ein Symposium unter der Schirmherrschaft des Cancer-Kontrollprogramms des Bundesgesundheitsamtes der Vereinigten Staaten von Nordamerika 81

Gall, S. A.; Bourgeois, C. H. und Maguire, R. (Fort Belvoir, Va.): Die mor-

phologische Auswirkung von Kontrazeptiva auf die Zervix 133

Garn, St. M.: vide Frisancho, A. R.

Gershowitz, H.: vide Solish, G. I.

Goldfien, A.: vide Friedman, St.

Gravelle, I. H.: vide Forrest, A. P. M.

Green, Th. H. und Morse, W. J. (Walpole, Mass.): Die Behandlung des invasiven

Kollumkarzinoms nach versehentlicher einfacher Hysterektomie 62

Greene, G. R.: vide Sartwell, P. E.

Gordon, G.: vide Emery, A. E. H.

Gryboski, J. D. (New Haven): Saug- und Schluckreflex beim Neugeborenen . . . 36

Hack, M.; Brish, M.; Serr, D. M.; Insler, V. und Lunenfeld, B. (Tel Aviv):

Ergebnis von Schwangerschaften nach Ovalutionsauslösung $\quad 30$

Hassim, A. M.: vide Lucas, C.

Heidrich, R. und Niedner, K. (Erfurt): Schwangerschaft und Subarachnoidal-

blutung 287

Hellman, L. M,; Dufuss, G. M.; Donald, J. und Sundén, B. (Brooklyn, N.Y.):

Sicherheit der Ultraschallanwendung in der geburtshilflichen Diagnostik . . 245 Hendee, W. R.:

vide Niehoff, R. D. Henderson, A.: vide Scott, J. M. Henk, E. N.: vide Forrest, A. P. M.

Hibbard,L. T. (Los Angeles, Cal.): Verhaken der Schulter an der Symphyse . . 293 Hirsh, J.;

Cade, J. F. und O'Sullivan, E. F.: Antikoagulantien-Therapie in der

Schwangerschaft 141

Hostrup-Petersen, J.: vide Fischerman, K.

Huvos, A. G.: vide Fracchia, A. A.

Hytten, F. E.: vide Paintin, D. B.

Insler, V.: vide Hack, M.

Irey, N. S.; Manion, W. C und Taylor, H. B. (Washington, D.C.): Vaskuläre

Läsionen nach Einnahme von Ovalutionshemmern 136 
Jain, V. C.: vide Prasad, B. G. Järvinen, P. A.: vide Luukkainen, T.

Inhaltsverzeichnis $\mathrm{V}$

Jurukovski, J. N. (Oxford): Komplikationen nach legalen Schwangerschaftsunterbrechungen 1

Kamat, M. H.; Del Gaizo, A. und Seebode, J. J. (Newark, NJ): Urethralprolaps

bei weiblichen Kindern $\quad 50$

Kaplan, N. M.: vide Martin, J. E.

Keeve, J. Ph.; Schlesinger, E. R.; Wight, B. W. und Adams, Ruth Ann (Pitts

burgh, Pa.): Schwangerschaften bei jungen Mädchen 199

Keith Mant, A. (London): Die Gefahren der legalen und illegalen Schwanger-

schaftsunterbrechung 3

Kerenyi, Th. D. (New York, N.Y.): Hypernatriämie nach intrauteriner Instillation

hypertoner Salzlösung295

Kircher, W. (Vöcklabruck): Zur Frage der unterschiedlichen Verteilung der ABO-

Blutgruppen bei erstgeborenen und nachgeborenen Kindern 120

Kneale, B. und Evans, J. (Melbourne) :Progestagen-Therapiebeim fortgeschrittenen

Karzinom des Endometriums 182

Koch, G. G.: vide Shrngleton, H. M.

Kratochwil, A. (Wien): Schädigungsmöglichkeiten durch Ultraschalldiagnostik? 240

Kraybill, E. N.: vide Avery, G. B.

Kurohara, S. S.: vide Badib, A. O.

Kvarnes, R. K.: vide Wenner, Naomi K.

Lauritzen, J. B.: vide Fischerman, K.

Lebherz, Th. B.: vide Mueller, M. J.

Leng, J. J.: vide Mahon, R.

Lucas, C. und Hassim, A. M. (Lusaka): Der Wert der Kuldozentese bei der ekto-

pischen Schwangerschaft 298

Lunenfeld, B.: vide Hack, M.

Luukkainen, T.; Väiströ, L. und Järvinen, P. A. (Helsinki): Die Wirkung von

peroral verabreichtem Alkohol auf die Motilität des schwangeren Uterus . . 210 Louden, L. M.:

vide Nash, E. M. MacDonald, P. C.: vide Martin, J. E. Macintosh, I. J. C. und Davay, D. A.

(Cape Providence): Chromosomenver-

änderungen, verursacht durch einen Pulsdetektor auf Ultraschallbasis $\quad 234$

MacMahon, B. und Austin, J. H. (Boston, Mass.): Kombination von Brustkrebs

und Korpuskarzinom 107

Magiure, R.: vide Gall, S. A.

Mahon, R.; Dubeco, J. P.; Leng, J. J. und Chenudet, P. (Bordeaux): Kann die

Thrombo-Embolie der Wöchnerin verhütet werden? 285

Mahran, M.: vide Fadel, H. E.

Mamunes, P.; Baden, M.; Bass, J. W. und Nelson, J. (Tacoma, Wash.): Frühe

intravenöse Ernährung bei Neugeborenen mit niederigem Geburtsgewicht . . . 223 Manion, W.

C.: vide Irey, N. S. Martin, J. E.; MacDonald, P. C. und Kaplan, N. M. (Dallas, Tex.): Erfolg-

reiche Schwangerschaft bei Sheehan-Syndrom 203

Masi, F. G.: vide Sartwell, P. E.

Maxwell, R. (New York): Probleme der Ovalutionsauslösung mit Comiphen, mit

einem Bericht über einen Fall von ovarieller Hyperstimulation 
VI

Inhaltsverzeichnis

McCann, St. W.; Mickal, A. und Crapanzano, T. J. (New Orleans, La.):

Scharfe Konisation der Zervix 174

McCartney, Ch. P. (Chicago, 111.): Hypertonie in der Schwangerschaft

McDonald, V. G.: vide Smith, A. I.

McNicol, G. P.: vide Bonnar, J.

McPherson, R. I.: vide Pusey, V. A.

Mickal, A.: vide McCann, St. W.

Morse, W. J.: vide Green, Th. H.

Motague, E. D.: vide Fletcher, G. H.

Müller-Heubach, E. (New Brunswick, N. J.): Hydronephrose bedingt durch

Uterusprolaps 229

Mueller, M. J., jr. und Lebherz, Th. B.: Thrombophlebitis in der Schwanger

schaft. Morbidítät bei Langzeitbehandlung mit Heparin - Ein Behandlungsvor-

schlag 274

Mullick, U. C.: vide Avery, G. B.

Nash, E. M. und Louden, L. M. (Winston-Salem, N.C.): Die medizinische Pflichtuntersuchung vor der Eheschliessung in Nordkarolina. Was die Patienten wünschen 308

Nelson, J.: vide Mamunes, P.

Nelson, M. M.: vide Emery, A. E. H.

$\mathrm{Ng}$, A.: vide Alan, B.

Niedner, K.: vide Heidrich, R.

Niehoff, R. D.; Hendee, W. R. und Brown, D. W. (Denver, Colo.): Plazenta-

szintigraphie mit In $113 \mathrm{~m} \quad 196$

Ohaneson, E. M.: vide Wenner, Naomi K.

O'Sullivan, E. F.: vide Hirsh, J.

Paintin, D. B.; Thomson, A. M. und Hytten, F. E. (Aberdeen): Eisen und Hämoglobinspiegel in der Schwangerschaft

Palo, A. J. De : vide Fracchia, A. A.

Palumbo, L.: vide Shingleton, H. M.

Patti, R. W.: vide Thomford, N. R.

Pearson, H. E. und Anderson, G. V. (Los Angeles, Calif.): Bacteroides-Infektion und Schwangerschaft 206

Peel, J. H.: vide Blacker, R. P.

Pigeaud, H. (Lyon): Blutungen in der Geburtshilfe infolge Ungerinnbarkeit des

Blutes. Möglichkeiten einer prophylaktischen Therapie 278

Podell, R. N. (Boston, Mass.): Schätzung der vermeidbaren Todesfälle an Brustkrebs 117

Prasad, B. G.; Srivastava, R. N.; Bhushan, Vidya und Jain, V. C. (Lucknow):

Untersuchungen über die jahreszeitliche Schwankung der Geburten in den ge-

burtshilflichen Kliniken einiger medizinischer Hochschulen in Indien 201

Prenzel, Claudia: vide Klaus, $\mathrm{K}$.

Pusey, V. A.; McPherson, R. I. und Chernick, V. (Winipeg): Fibropasie der

Lungen bei Neugeborenen nach längerdauernder künstlicher Beatmung .... 40 
Raby, C. und Bertrand, H. (Paris): Dextrane und Dynamik der Blutgerinnung . . 148

Reagan, J. W.: vide Alan, B.

Inhaltsverzeichnis VII

Reyniak, J. V.; Sedlis, A.; Stone, D.; Connell, E. (New York, N.Y.): Funktions-

zytologische Befunde bei Patientinnen unter verschiedenen Kontrazeptiva . . . 312 Richardson, F.

H. und Stonington, O. G. (Boulder, Colo.): Urethrolyse und

äussere Urethroplastik bei der Frau 303

Rohmann, Ch. G.: vide Frisancho, A. R.

Sabour, M. S.: vide Fadel, H. E.

Sartwell, P. E.; Masi, F. G.; Arthes, F. G.; Greene, G. R. und Smith, H. E.

(Baltimore, MD): Thromboembolie und orale Contraceptiva 68

Sayre, J. E.: vide Cohen, B. H.

Schlesinger, E. R.: vide Keeve, J. Ph.

Scott, J. M. und Henderson, A. (Glasgow): Ein Fall von Listeriose beim Neuge-

borenen $\quad 43$

Sedlis, A.: vide Reyniak, J. V.

Seebode, J. J.: vide Kamat, M. H.

Seif El-Din, D.: vide Fadel, H. E.

Serr, D. M.: vide Hack, M.

Shingleton, H. M.; Fowler, W. C; Palumbo, L. und Koch, G. G. (Chapel Hill,

N.C.): Der Einfluss der Radikaloperation auf die Heilungsrate des Vulva-

karzinoms 171

Smith, A. I. und McDonald, V. G. (Kansas City, MO): Schwierigkeiten bei der

Chirurgie des weiblichen Genitalapparates im Kindesalter 52

Smith, H. E.: vide Sartwell, P. E.

Smith, J. P.: vide Antolak, S. J., jr.

Solish, G. I. und Gershowitz, H. (Brooklyn, N.Y.): Die Verteilung der ABO-

Blutgruppen unter fertilen und infertilen Frauen 130

Steptoe, P. C. (Oldham): Fortschritte in den chirurgischen Methoden zur Kontrolle

der Fertilität und Infertilität 9

Stone, D.: vide Reyniak, J. V.

Stonington, O. G.: vide Richardson, F. H.

Srivastava, R. N.: vide Prasad, B. G.

Sussmann, M.; Asscher, A. W.; Waters, W. E.; Evans, J. A. S.; Campbell, H.;

Evans, K. T. und Williams, J. E. (Cardiff): Starke asymptomatische Bakteri-

urie bei nichtschwangeren Frauen 301

Sundén, B.: vide Hellman, L. M.

Taylor, H. B.: vide Irey, N. S.

Teokharov, B. A. (Omsk): Nicht durch Gonokokken bedingte Infektionen der

weiblichen Genitalorgane 315

Teteris, N. J.: vide Thomford, N. R.

Teubner, D.: vide Florman, A. L.

Thomford, N. R.; Patti, R. W. und Teteris, N. J. (Columbus, Ohio): Appendek-

tomie während der Schwangerschaft 289

Thomson, A. M.: vide Paintin, D. B.

Tong, D. (London): Die Behandlung solitärer Zysten in der Mamma: eine neue 
Technik $\quad 98$

Väiströ, L.: vide Luukkainen, T. Vauclair, R.: vide Audet-Lapointe, P.

VIII

Inhaltsverzeichnis

Voigt, J. C. und Britt, R. P. (Uxbridge): Die feto-maternale Hämorrhagie bei

Schwangerschaftsunterbrechungen 5

Waters, W. E.: vide Sussmann, M.

Webster, J. H.: vide Badib, A. O.

Weigert, E. V.: vide Wenner, Naomi K.

Wenner, Naomi K.; Cohen, M. B.; Weigert, E. V.; Kvarnes, R. K.; Ohaneson,

E. M. und Fearing, J. M. (Washington, Wash.): Affektive Problematik in der

Schwangerschaft 192

White, C.: vide Wyshak, G.

White, E. C.: vide Fletcher, G. H.

Wight, B. W.: vide Keeve, J. Ph.

Williams, J. E.: vide Sussmann, M.

Wyshak, G. und White, C. (New Haven, Conn.): Die Fertilität von Zwillingen und deren Verwandten 198

Zippin, C.: vide Cutler, S. J.

Kasuistik

Babenerd, J. (Würzburg): Eine primäre Abdominalgravidität

Linka, F. (Würzburg): Missbildung des Vorderhauptes und Mikromelie bei mehreren teratogenen Einflüssen 73

AUS DER GESCHICHTE DER GEBURTSHILFE

Klaus, K. (Prag) und Prenzel, Claudia (Basel): Franz Alexander Wilhelm Kiwisch

«Ritter von Rotterau» (1814-1851) 155

Varia

3. Internationaler Kongress für Psychosomatische Medizin in Geburtshilfe und

Gynäkologie, März 1971 London 154

Sonderbeilage

Kann die Ultraschallüberwachung den Fetus schädigen?

233

Prüfungsfragen für Fachärzte

Prüfungsfragen $\quad 79,159,231,319$

Richtige Antworten der Fragen $\quad 80,160,232,320$

S. Karger · Basel - München · New York Arnold-Böcklin-Strasse 25, CH-4000 Basel 11 (Schweiz)

Alle Rechte, insbesondere das der Übersetzung in andere Sprachen, vorbehalten.

Ohne ausdrückliche Genehmigung des Verlages ist es auch nicht gestattet,

diesen Band Oder Teile daraus auf photomechanischem Wege (Photokopie, Mikrokopie) zu

vervielfältigen

(C)

Copyright 1970 by S. Karger AG, Verlag für Medizin und Naturwissenschaften, Basel

Printed in Switzerland by Buchdruckerei National-Zeitung, Basel

Klischees: Steiner \& Co. AG, Basel 